(6) OPEN ACCESS

\title{
Is there evidence that walking groups have health benefits? A systematic review and meta-analysis
}

\author{
Sarah Hanson, Andy Jones
}

- Additional material is published online only. To view please visit the journal online (http://dx.doi.org/10.1136/ bjsports-2014-094157).

Norwich Medical School, University of East Anglia, Norwich, Norfolk, UK

\section{Correspondence to} Sarah Hanson, Norwich Medical School, University of East Anglia, Norwich, Norfolk NR4 7TJ, UK s.hanson@uea.ac.uk

Received 22 August 2014 Revised 21 October 2014 Accepted 29 November 2014 Published Online First 19 January 2015

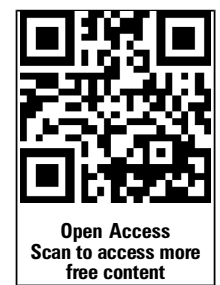

CrossMark

To cite: Hanson $S$, Jones $A$. $\mathrm{Br} J$ Sports Med

2015:49:710-715

\section{ABSTRACT}

Objective To assess the health benefits of outdoor walking groups.

Design Systematic review and meta-analysis of walking group interventions examining differences in commonly used physiological, psychological and well-being outcomes between baseline and intervention end.

Data sources Seven electronic databases, clinical trial registers, grey literature and reference lists in English language up to November 2013.

Eligibility criteria Adults, group walking outdoors with outcomes directly attributable to the walking intervention.

Results Forty-two studies were identified involving 1843 participants. There is evidence that walking groups have wide-ranging health benefits. Meta-analysis showed statistically significant reductions in mean difference for systolic blood pressure $-3.72 \mathrm{~mm} \mathrm{Hg}$ $(-5.28$ to -2.17$)$ and diastolic blood pressure $-3.14 \mathrm{~mm} \mathrm{Hg}(-4.15$ to -2.13$)$; resting heart rate -2.88 bpm ( -4.13 to -1.64$)$; body fat $-1.31 \%$ $(-2.10$ to -0.52$)$, body mass index $-0.71 \mathrm{~kg} / \mathrm{m}^{2}$ $(-1.19$ to -0.23$)$, total cholesterol $-0.11 \mathrm{mmol} / \mathrm{L}$ $(-0.22$ to -0.01$)$ and statistically significant mean increases in $\mathrm{VO}_{2 \max }$ of $2.66 \mathrm{~mL} / \mathrm{kg} / \mathrm{min}(1.67-3.65)$, the SF-36 (physical functioning) score 6.02 (0.51 to 11.53 ) and a 6 min walk time of $79.6 \mathrm{~m}$ (53.37-105.84). A standardised mean difference showed a reduction in depression scores with an effect size of $-0.67(-0.97$ to -0.38 ). The evidence was less clear for other outcomes such as waist circumference fasting glucose, SF-36 (mental health) and serum lipids such as highdensity lipids. There were no notable adverse side effects reported in any of the studies.

Conclusions Walking groups are effective and safe with good adherence and wide-ranging health benefits. They could be a promising intervention as an adjunct to other healthcare or as a proactive health-promoting activity.

\section{INTRODUCTION}

Regular physical activity positively impacts health potentially offering similar effects to some drug interventions in terms of mortality benefits. Indeed, it has been suggested as an alternative or adjunct to conventional drug therapy. ${ }^{1}$ Walking at a pace of 3$5 \mathrm{~m} / \mathrm{h}(5-8 \mathrm{~km} / \mathrm{h})$ expends sufficient energy to be classified as moderate intensity ${ }^{2}$ and is an easy and accessible way of meeting physical activity recommendations. ${ }^{3}$ Systematic reviews and meta-analyses have shown walking to have various health benefits including positive effects on fitness, fatness and resting blood pressure, ${ }^{4}$ blood pressure control, ${ }^{5}$ weight loss, ${ }^{6}$ depression ${ }^{7}$ and cardiovascular disease risk prevention. ${ }^{8}$
Despite evidence and government campaigns such as Change4life ${ }^{9}$ to promote physically active lifestyles, few are active enough to be of benefit to general health. In England, for example, 29\% of adults do less than $30 \mathrm{~min}$ of moderate physical activity per week ${ }^{10}$ and about $8 \%$ do not even walk continuously for 5 min over 4 weeks. ${ }^{11}$ The impact of interventions in primary care to reduce inactivity appears limited; simple advice to be more active has only moderate yet short-term effects and an effective way of increasing physical activity and improving associated health indicators while also making the most efficient use of doctors' resources has yet to be determined. ${ }^{12-14}$

One way to promote and sustain walking behaviours at the population level may be through the provision of outdoor walking groups. ${ }^{15}$ Walking groups are typically short walks of under an hour in the natural environment, run by trained lay people. An example of such is 'Walking for Health', a scheme originally set up by an Oxford General Practitioner in 2000. It is England's largest network of lay-led health group walks with 70000 regular walkers, 10000 volunteer walk leaders and approximately 3000 short walks offered every week. ${ }^{16}$ Group walking is a potentially attractive physical activity intervention that has particular potential to engage those who are interested in the outdoors, whether for leisure or as a health intervention and has been found to be cost-effective in increasing physical activity. ${ }^{17}$ Additionally, the dynamics and social cohesion of walking groups may have supportive effects that encourage and sustain adherence and positive attitudes towards physical activity, ${ }^{18}$ companionship and a shared experience of wellness. ${ }^{19}$ A systematic review in 2007 by Ogilvie et $a l^{20}$ concluded that people could be encouraged to walk more if interventions were tailored to their needs and targeted at the most sedentary or at those most motivated to change and that group-based approaches, such as the social support of walking groups, are one method of delivering this. In a recent review, walking groups were found to be efficacious at increasing physical activity, particularly when targeted at older adults. ${ }^{21}$ However, it remains that the benefits to health from increasing physical activity are greater than increasing fitness levels, yet no review to date has attempted to quantify the wider health benefits of walking groups. Hence, this review has been undertaken to understand whether there is evidence that outdoor walking groups show wider health benefits as an intervention and therefore could be recommended by clinicians.

\section{METHODS}

This systematic review followed Cochrane systematic review guidelines, ${ }^{22}$ requirements of the NHS National Institute of Health Research Centre for 
Reviews and Dissemination ${ }^{23}$ and the PRISMA statement for reporting studies that evaluate healthcare interventions. ${ }^{24} 25$ Methods of the analysis and inclusion criteria were specified in advance and documented in a protocol registered as CRD42013006397 ${ }^{23}$ available at http://www.crd.york.ac.uk/ prospero/

\section{Data sources}

We searched using electronic databases; clinical trials registers; by scanning reference lists of articles and from grey literature. For the electronic databases, the search with specific search terms was applied in to AMED, EMBASE, MEDLINE (R) in process and other non-indexed citations and PsycINFO (sourced through OVID); SportDiscus and CINAHL (sourced through EBSCO) and SCOPUS with no date restriction. Databases were selected to best represent source material in health, allied health, physical activity and human science. Clinical trials registers were searched through the UK clinical trials research network study portfolio; clinicaltrials.gov and controlledtrials. com. Grey literature included reports from Natural England, Walking for Health and the National Institute for Health and Care Excellence. Additionally, reference lists from included studies and systematic reviews on exercise and walking were hand searched. The search was completed in November 2013.

Inclusion criteria were studies of outdoor walking groups involving adults with measured physiological, psychological or well-being outcomes. The search was restricted to papers published in English. The inclusion criteria are further detailed in table 1.

Search terms were developed with reference to the previous systematic reviews on walking ${ }^{20}$ and key words from relevant studies. They were piloted to ensure that known studies were identified. The search syntax for the electronic databases is detailed in online supplementary information appendix (i). For clinical trials registers, the only search term was 'walking' within the title.

\section{Study selection}

All studies where the outcome could be directly attributable to the group walking were included. This included studies where walking was the control group. All studies were reviewed by the first reviewer and duplicates or the clearly irrelevant, for example, walk-in centres, using wii-fit, or studies using children or animals that had not been screened out by the database filters were excluded. A particular issue with the assessment of the studies was that the phrase 'walking group' often related to a walking arm of a study, or a group within a trial that could walk, and not a 'walking group' per se. Additionally, there was commonly little information within the abstract about the setting of the intervention, for example, treadmill or indoor circuit-based interventions or home-based solo interventions with physical activity diaries and pedometers. Therefore, most studies were retrieved as full texts and scanned for intervention information to ensure that none were excluded incorrectly. Owing to the generally poor description of the intervention, 40 authors were contacted to confirm whether the study was an outdoor intervention and that they walked as a group. To further ensure that studies had been correctly excluded, $15 \%$ of the excluded studies were selected by random number generation and screened by the second author (AJ). All papers were found to have been excluded correctly and therefore no further excluded studies were reviewed.

\section{Data extraction}

A data extraction sheet was developed by both authors to summarise the study, the population, walking group characteristics, the intervention (volume and intensity), adherence and outcomes. This was piloted on five manuscripts and refined accordingly. Data were extracted by the first reviewer into a coding frame using Microsoft Excel, synthesised and tabulated.

\section{Risk of bias in individual studies and across studies within meta-analyses}

As not all studies were randomised controlled trials, a tool used by Ogilvie et $a l^{20}$ was adapted to assess risk of bias and internal validity ${ }^{26}$ with nine items on a binary scale. These were:

1. Randomisation: Was there sufficient description of a randomisation process or statistical test to show that comparability between the two groups has been adjusted for (no explanation scores scored zero)?

2. Exposure: Did the authors show that there was no evidence of a concurrent intervention which could have influenced the results (no explanation scores zero)?

Table 1 Inclusion and exclusion criteria

Inclusion
Adults from the age of 19
Interventions where people walk as part of a defined walking group intervention

Where the walking is group based, or where the walking is predominantly group based but participants may also walk on their own to supplement this Walking outdoors or walking predominantly outdoors but occasionally indoors (eg, inside tracks or shopping malls for weather reasons)

Studies that compare group walking with group Nordic walking where group walking can be isolated as an intervention and the outcome directly related to group walking

Studies with physiological, psychological or well-being outcomes such as blood profiles (eg, lipids, HbA1c), cardiovascular measures (eg, BP), psychological (eg, Beck depression inventory), well-being (eg, EQ5D)

Studies where the outcome can directly be related to the walking group intervention

Papers and documents written in English

\section{Exclusion}

Youths and children up to and including 18

Studies that do not involve a walking group intervention, eg, they walk with a physiotherapist

Participants walking only rarely in groups, or walking on their own, such as home-based or pedometer-based programmes with no group walking

Walking indoors or predominantly indoors

Studies examining Nordic walking only

Studies where the outcomes are solely physical activity such as step outcomes or logs of physical activity

Studies with a mixed intervention (eg, walking with calcium supplements or walking combined with a health education intervention) where the outcome cannot be isolated and directly attributed to group walking

Papers and documents not written in English 
3. Representativeness: Were the study samples shown to be representative of the study population?

4. Comparability: Were baseline characteristics of the intervention comparable with the control or were potential confounders at baseline appropriately adjusted for in analysis?

5. Attrition: Were numbers of participants at follow-up identifiable as at least $80 \%$ of the baseline?

6. Follow-up tools: Were valid and reliable tools used to assess participant outcomes?

7. Follow-up time scale: Was the time to follow-up assessment of a period no less than 1 month?

8. Precision of the results: Were CIs or $\mathrm{p}$ values given?

9. Was there evidence presented that the study was sufficiently powered at follow-up assessment (no evidence or underpowered scores zero)?

Publication bias across studies within the meta-analysis was tested with funnel plots using SE as the measure of study size on the vertical axis ${ }^{27}$ and mean difference on the horizontal.

\section{Synthesis of results and statistical analysis}

Data for the final studies were synthesised with results for each study recorded as change from baseline to the end of the intervention $(\uparrow \downarrow)$ with $\mathrm{p}$ values where available. Non-significant or imprecise $p$ values, such as $p>0.05$, were used only when this was the only available information. No assumptions were made about walking outside the group provision. To establish the mean difference between baseline and the end of intervention for meta-analysis, baseline data with SD and sample size, and end of intervention data with SD or SE and sample size were utilised. All data were continuous and a difference in means was used except for one analysis; for depression a standardised mean difference was used to account for the different outcome measurements used in the five studies. There was no need for data to be transformed as a reduction in value indicated an improvement in health in all four outcome measures within this analysis. A fixed effects model was used for all analyses representing a more conservative measure than a random effects model. ${ }^{22}$ Where data were given for different subgroups, each was input separately and combined in meta-analyses using the RevMan software package. ${ }^{28}$ All results are presented with 95\% CIs. The $\mathrm{I}^{2}$ statistic was used to test for heterogeneity. $\mathrm{I}^{2}$ values of $30-60 \%$ and $50-90 \%$ were taken to represent moderate and substantial heterogeneity, respectively (ref. 22, Ch 9.5.2).

\section{RESULTS}

The initial database search yielded 5145 citations. In addition, the other supplementary sources produced a further 60 studies. Of these 5205 studies, 4627 were removed as duplicates or as clearly irrelevant after reviewing titles. The abstracts of 578 articles were screened and any that did not provide enough information were retrieved for full-text evaluation. A total of 150 papers were read as full texts to be assessed for eligibility. The remaining 46 articles were put forward for second review and independently assessed by the second reviewer (AJ). From this, 10 papers were discussed between the two reviewers. Three studies were excluded due to a lack of information despite repeated attempts to contact authors as the reviewers lacked confidence that the intervention was group based and outdoors. One was excluded on further discussion due to the walking being primarily self-directed. In total, 42 studies met the inclusion criteria and were eligible to be included in the synthesis. Walking groups were used as a control in seven of the studies. The review flow chart is detailed in online supplementary figure S1. The characteristics and synthesised results from all 42 studies are detailed in online supplementary table S1.

All 42 studies were assessed for risk of bias (table 2). No study was excluded due to a low-quality score. Assessments of quality were made by the first reviewer and $20 \%$ of the studies were chosen by random number generation and checked by the second reviewer. An inter-rater reliability analysis using the $\kappa$ statistic was performed to determine consistency among raters and found to be $\kappa 0.66(\mathrm{p}<0.001)$ representing substantial agreement.

\section{Study characteristics}

Although there was no date restriction on the search, $74 \%$ of the articles were studies in the past 10 years suggesting the recent interest in walking groups, with no papers prior to 1988 meeting the inclusion criteria. Studies were located in 14 different countries but predominantly in the USA $(n=15)$. A total of 1843 participants walked in outdoor walking groups with at least $1488 \mathrm{~h}$ of provision (3 studies did not give enough information from which to calculate dosage) and a total of $74023 \mathrm{~h}$ of participant walking time. Walking groups were used with participants with a broad range of health conditions: arthritis, ${ }^{29} 30$ dementia and cognitive impairment, ${ }^{31-33}$ diabetes, ${ }^{34-36}$ fibromyalgia, ${ }^{37-39}$ obesity and overweight, ${ }^{40-44}$ mental health issues $^{45-49}$ and Parkinson's disease ${ }^{50}$ with 64 different tools used to test outcomes.

In terms of participants, $76 \%$ were women while $43 \%$ of the studies were for women only; there were no studies for men only. The grand mean age was 58 years with 15 studies specifically aimed at older participants. There was subanalysis in four studies: ethnicity, ${ }^{40}$ intensity $^{47}{ }^{51}$ and gender. ${ }^{43}$ Two studies were of people with learning disabilities living in care facilities: one obese adults with Prader-Willi syndrome, ${ }^{52}$ and the second the coronary heart disease risk of adults with learning disabilities. ${ }^{43}$ Eleven studies described the ethnicity of the participants and 13 studies provided some socioeconomic information. Brandon and Elliott-Lloyd ${ }^{40}$ compared the response between African-American women, and the O'Hara et $a l^{44}$ study was specifically for African-American women. Otherwise there was no evaluation of effect for different ethnicities.

Interventions were varied, in volume and intensity, ranging from 168 to $8580 \mathrm{~min}$ of walking over a period of 3 weeks to 1 year, with intensity ranging from self-selected and low to brisk walking and high-intensity intervals. Moore-Harrison et al ${ }^{53}$ specifically targeted those of low socioeconomic profile, and Isaacs et $a l^{54}$ provide subanalysis of uptake of walking group intervention by socioeconomic status. Where supervision was described, it was by professionals, such as physiotherapists, possibly as the interventions were part of clinical trials. Where described, provision was in rural locations in 6 of the studies, and urban for 15 . Where additional information from authors has been obtained, this has been added to the results table 2 (see online supplementary information). Adherence and adverse effects are described in $76 \%$ of the papers. Mean adherence (where stated) was $75 \%$. One study notes that adherence was lower for those without access to private transport. ${ }^{54}$ For adverse effects, one study described one fall with a brief absence from the walking programme, ${ }^{55}$ one a calf injury ${ }^{46}$ and one, a study with participants with Parkinson's disease, describes one participant experiencing exercise-induced hypotension after intense uphill walking in hot weather and four falls on roots and wet ground. ${ }^{50}$ Otherwise, either authors state that there were no injuries, or there is no reference to adverse effects. This is against a back drop of over 74000 participant hours. 
Table 2 Risk of bias for included studies

\begin{tabular}{|c|c|c|c|c|c|c|c|c|c|c|c|}
\hline \multirow[b]{2}{*}{ Author } & \multirow[b]{2}{*}{ Study type } & \multicolumn{9}{|c|}{ Risk of bias items } & \multirow[b]{2}{*}{ Total score } \\
\hline & & 1 & 2 & 3 & 4 & 5 & 6 & 7 & 8 & 9 & \\
\hline Armstrong & RCT & 1 & 0 & 1 & 1 & 1 & 1 & 1 & 0 & 1 & 7 \\
\hline Bjersing & RCT & 1 & 0 & 1 & 1 & 1 & 1 & 1 & 1 & 0 & 7 \\
\hline Brandon & RCT & 0 & 1 & 1 & 1 & 1 & 1 & 1 & 1 & 1 & 8 \\
\hline Brosseau & RCT & 1 & 0 & 1 & 1 & 0 & 1 & 1 & 1 & 0 & 6 \\
\hline Cox & RCT & 1 & 1 & 1 & 1 & 1 & 1 & 1 & 1 & 1 & 9 \\
\hline Duncan & $\mathrm{RCT}$ & 0 & 1 & 1 & 1 & 1 & 1 & 1 & 1 & 0 & 7 \\
\hline Fisher & RCT & 1 & 0 & 1 & 1 & 0 & 1 & 1 & 1 & 0 & 6 \\
\hline Gusi & RCT & 1 & 0 & 1 & 1 & 1 & 1 & 1 & 1 & 1 & 8 \\
\hline Hamdorf & RCT & 1 & 1 & 1 & 1 & 0 & 1 & 1 & 1 & 0 & 7 \\
\hline Hinkleman & RCT & 0 & 1 & 1 & 1 & 0 & 1 & 1 & 1 & 0 & 6 \\
\hline Isaacs & RCT & 1 & 1 & 1 & 1 & 1 & 1 & 1 & 1 & 1 & 9 \\
\hline Kamijo & RCT & 0 & 1 & 1 & 1 & 1 & 1 & 1 & 1 & 0 & 7 \\
\hline Kayo & RCT & 1 & 0 & 1 & 1 & 0 & 1 & 1 & 1 & 1 & 7 \\
\hline Legrand & RCT & 0 & 0 & 1 & 1 & 1 & 1 & 1 & 1 & 0 & 6 \\
\hline Mannerkorpi & RCT & 1 & 1 & 1 & 1 & 0 & 1 & 1 & 1 & 1 & 8 \\
\hline Moore-Harrison & RCT & 0 & 0 & 1 & 1 & 1 & 1 & 1 & 1 & 0 & 6 \\
\hline Morrison & RCT & 0 & 0 & 1 & 1 & 1 & 1 & 1 & 1 & 0 & 6 \\
\hline Negri & RCT & 1 & 0 & 1 & 1 & 0 & 1 & 1 & 1 & 0 & 6 \\
\hline Palmer & RCT & 0 & 1 & 1 & 1 & 1 & 1 & 1 & 0 & 0 & 6 \\
\hline Reuter & RCT & 1 & 1 & 1 & 1 & 1 & 1 & 1 & 1 & 1 & 9 \\
\hline Rooks & RCT & 1 & 1 & 1 & 1 & 1 & 1 & 1 & 1 & 0 & 8 \\
\hline van Uffelen & $\mathrm{RCT}$ & 1 & 0 & 1 & 1 & 1 & 1 & 1 & 1 & 0 & 7 \\
\hline Callahan & Pre-post & 0 & 0 & 1 & 1 & 1 & 1 & 1 & 1 & 0 & 6 \\
\hline Dallocchio & Pre-post & 0 & 0 & 1 & 1 & 1 & 1 & 1 & 1 & 0 & 6 \\
\hline Fantin & Pre-post & 0 & 0 & 1 & 1 & 1 & 1 & 1 & 1 & 1 & 7 \\
\hline Figard-Fabre & Pre-post & 0 & 1 & 1 & 1 & 1 & 1 & 1 & 1 & 0 & 7 \\
\hline Gelecek & Pre-post & 0 & 1 & 1 & 1 & 1 & 1 & 1 & 0 & 0 & 6 \\
\hline Holmberg & Pre-post & 0 & 0 & 1 & 1 & 0 & 1 & 1 & 1 & 0 & 5 \\
\hline Moss & Pre-post & 0 & 0 & 1 & 1 & 1 & 1 & 1 & 0 & 0 & 5 \\
\hline O'Halloran & Pre-post & 0 & 0 & 1 & 1 & 1 & 1 & 1 & 0 & 0 & 5 \\
\hline O'Hara & Pre-post & 0 & 0 & 1 & 0 & 1 & 1 & 1 & 0 & 0 & 4 \\
\hline Cavanaugh & CT & 0 & 1 & 1 & 1 & 1 & 1 & 1 & 1 & 0 & 7 \\
\hline Fritz & CT & 0 & 0 & 1 & 0 & 1 & 1 & 1 & 1 & 0 & 5 \\
\hline Park & CT & 0 & 1 & 1 & 1 & 0 & 1 & 1 & 1 & 0 & 6 \\
\hline Roberts & CT & 0 & 1 & 1 & 1 & 1 & 1 & 1 & 0 & 0 & 6 \\
\hline Silverthorn & $\mathrm{CT}$ & 0 & 1 & 1 & 1 & 1 & 1 & 1 & 0 & 0 & 6 \\
\hline Song & CT & 1 & 0 & 1 & 1 & 1 & 1 & 1 & 1 & 1 & 8 \\
\hline Takahashi & CT & 0 & 1 & 0 & 1 & 1 & 1 & 1 & 1 & 0 & 6 \\
\hline Thomas & $\mathrm{CT}$ & 0 & 0 & 1 & 1 & 1 & 0 & 1 & 0 & 0 & 4 \\
\hline Cyarto & Quasi-experimental & 0 & 1 & 1 & 1 & 1 & 1 & 1 & 1 & 0 & 7 \\
\hline McDevitt & Quasi-experimental & 0 & 0 & 1 & 1 & 1 & 1 & 1 & 1 & 0 & 6 \\
\hline $\mathrm{Ng}$ & Cohort study & 0 & 0 & 1 & 0 & 1 & 1 & 1 & 0 & 0 & 4 \\
\hline
\end{tabular}

(1) Randomisation, (2) exposure, (3) representativeness, (4) comparability, (5) attrition, (6) follow-up tools, (7) follow-up time scale, (8) precision of the results, (9) statistical power. Grey scale indicates studies included in meta-analysis.

$\mathrm{CT}$, controlled trial; RCT, randomised controlled trial.

Attrition was less clearly described but in one study there was a participant withdrawal as overweight and self-conscious; ${ }^{45}$ one author states that travel to the walking club may have affected attrition, ${ }^{29}$ and one describes the different attrition rates between African-American and white walkers. ${ }^{40}$

\section{Meta-analysis}

Common outcome measures enabled meta-analysis of 17 frequently used outcome measures, summarised in table 3 and presented in full in online supplementary information appendix (iii). Statistically significant improvements from baseline to end of intervention were identified for participants in the intervention groups for systolic and diastolic blood pressure, resting heart rate, body fat, body mass index (BMI), total cholesterol, $\mathrm{VO}_{2 \max }$, quality of life for physical functioning, $6 \mathrm{~min}$ walk time and depression. For depression, a standardised mean difference of $-0.67(-0.97$ to -0.38$)$ represents a statistically significant moderate effect. $^{22}$ For other outcomes, the effects were not statistically significant.

There was zero heterogeneity in 12 of the analyses with 4 having an $\mathrm{I}^{2}$ between $28 \%$ and $48 \%$. The depression score had an $\mathrm{I}^{2}$ of $83 \%$ suggesting a high level of heterogeneity between the studies. Using funnel plots, all studies were visually symmetrical with a narrow spread at the top of the funnel indicating precision 
Table 3 Summary meta-analysis results table: difference between baseline and end of intervention

\begin{tabular}{|c|c|c|c|c|c|}
\hline Outcome measure & n & Effect & $95 \% \mathrm{Cls}$ & Heterogeneity & Test for overall effect \\
\hline Systolic BP (mm Hg) & 440 & -3.72 & $(-5.28$ to -2.17$)$ & $\chi^{2}=12.02, d f=12(p=0.44) ; l^{2}=0 \%$ & $z=4.70(p<0.001)$ \\
\hline Diastolic BP (mm Hg) & 440 & -3.14 & $(-4.15$ to -2.13$)$ & $\chi^{2}=23.16, d f=12(p=0.03) ; l^{2}=48 \%$ & $z=6.09(p<0.001)$ \\
\hline Resting HR (bpm) & 252 & -2.88 & $(-4.13$ to -1.64$)$ & $\chi^{2}=2.96, d f=7(p=0.89) ; l^{2}=0 \%$ & $z=4.53(p<0.001)$ \\
\hline Body fat (\%) & 328 & -1.31 & $(-2.10$ to -0.52$)$ & $\chi^{2}=4.00, d f=6(p=0.68) ; l^{2}=0 \%$ & $z=3.25(p=0.001)$ \\
\hline Body mass index $\left(\mathrm{kg} / \mathrm{m}^{2}\right)$ & 451 & -0.71 & $(-1.19$ to -0.23$)$ & $\chi^{2}=5.52, d f=11(p=0.90) ; l^{2}=0 \%$ & $z=2.92(p=0.003)$ \\
\hline Total cholesterol $(\mathrm{mmol} / \mathrm{L})$ & 271 & -0.11 & $(-0.22$ to -0.01$)$ & $\chi^{2}=12.58, d f=9(p=0.18) ; l^{2}=28 \%$ & $z=2.13(p=0.03)$ \\
\hline $\mathrm{VO}_{2 \max }(\mathrm{mL} / \mathrm{kg} / \mathrm{min})$ & 166 & 2.66 & (1.67 to 3.65$)$ & $\chi^{2}=9.67, d f=6(p=0.14) ; 1^{2}=38 \%$ & $z=5.28(p<0.001)$ \\
\hline SF-36 score (physical functioning) (points) & 68 & 6.02 & (0.51 to 11.53$)$ & $\chi^{2}=0.26, d f=1(p=0.61) ; l^{2}=0 \%$ & $z=2.14(p=0.03)$ \\
\hline 6 min walk time $(\mathrm{m})$ & 65 & 79.6 & (53.37 to 105.84 ) & $\chi^{2}=0.71, d f=1(p=0.40) ; l^{2}=0 \%$, & $z=5.95(p \leq 0.001)$ \\
\hline Depression score* (effect size) & 101 & -0.67 & $(-0.97$ to -0.38$)$ & $\chi^{2}=24.14, d f=4(P \leq 0.001) ; I^{2}=83 \%$ & $z=4.44(p \leq 0.001)$ \\
\hline Waist circumference $(\mathrm{cm})$ & 35 & -3.55 & $(-8.08$ to 0.98$)$ & $\chi^{2}=0.52, d f=1(p=0.47) ; l^{2}=0 \%$ & $z=1.54(p=0.12)$ \\
\hline $\mathrm{HbA1c}(\%)$ & 66 & -0.11 & $(-0.25$ to 0.03$)$ & $\chi^{2}=1.17, d f=3(p=0.76) ; l^{2}=0 \%$ & $z=1.53(p=0.13)$ \\
\hline Fasting glucose (mmol/L) & 85 & -0.09 & $(-0.28$ to 0.11$)$ & $\chi^{2}=3.33, d f=4(p=0.50) ; l^{2}=0 \%$ & $\mathrm{z}=0.87(\mathrm{p}=0.38)$ \\
\hline Low-density lipids (mmol/L) & 268 & -0.05 & $(-0.16$ to 0.06$)$ & $\chi^{2}=8.83, d f=9(p=0.45) ; l^{2}=0 \%$, & $z=0.93(p=0.35)$ \\
\hline High-density lipids (mmol/L) & 251 & 0.01 & $(-0.04$ to 0.07$)$ & $\chi^{2}=8.04, d f=8(p=0.43) ; l^{2}=0 \%$ & $z=0.45(p=0.65)$ \\
\hline Triglycerides (mmol/L) & 271 & -0.05 & $(-0.12$ to 0.03$)$ & $\chi^{2}=13.39, d f=9(p=0.15) ; l^{2}=33 \%$ & $z=1.25(p=0.21)$ \\
\hline SF-36 score (mental health index) (points) & 68 & 2.70 & $(-2.09$ to 7.48$)$ & $\chi^{2}=0.18, d f=1(p=0.67) ; I^{2}=0 \%$ & $z=1.10(p=0.27)$ \\
\hline
\end{tabular}

${ }^{*}$ All analyses fixed effects model and mean difference except depression score (effect is standardised mean difference).

$\mathrm{BP}$, blood pressure; HbA1c, glycated haemoglobin; HR, heart rate.

with results close to the pooled estimate and without bias towards smaller studies (see online supplementary appendix ii).

In order to test if the impact of the group walking was greater in those with clearly defined morbidity, a sub analysis was completed for the conditions of overweight or obese (BMI $\geq 25)$, Type II diabetes (as defined by authors) and depression (as defined by authors). For depression and BMI this strengthened the results. By only including those defined as depressed ${ }^{17} 4547$ the effect size became large $-0.76(-1.12$ to -0.41$)$. By only including those with a BMI $\geq 25^{17} 34 \quad 35404143 \quad 54 \quad 5657$ the mean difference increased to $-0.75(-1.26$ to -0.24$)$. For glycated haemoglobin (HbA1c) and fasting glucose, only including those with type II diabetes ${ }^{34}$ the mean differences remained statistically not significant $-0.16(-0.40$ to 0.08$)$ and -0.57 $(-1.58$ to 0.43$)$ respectively.

\section{DISCUSSION \\ Principal findings}

This systematic review and meta-analysis provides evidence that outdoor walking groups have health benefits over and above making people more physically active. Statistically significant improvements were found in a range of widely used measures of health; systolic and diastolic blood pressure, resting heart rate, body fat, $\mathrm{BMI}$, total cholesterol, $\mathrm{VO}_{2 \max }$, depression, 6-min walk time, and quality of life for physical functioning. This is despite the fact that the majority of the interventions (75\%) were below international moderate activity guidelines which may account for some of the effect sizes being small. Walking groups appear an acceptable intervention to participants with high levels of adherence and a low risk of serious adverse effects.

\section{Strengths and limitations}

The strength of this review is that it has comprehensively sought out walking group studies. It has extensively analysed 42 different studies with 1843 participants involved in over 74000 participant hours of group walking. It has also extracted information for 17 meta-analyses to provide evidence of health benefits and within these was generally zero or low heterogeneity. Limitations of the study are that only manuscripts published in English were sought. Additionally, the populations in the included studies are very different with many small studies. The lack of information on walking dose in many of the studies mean it was not possible to undertake an analysis of dose-responses.

\section{Results in context of other published reviews}

Kassavou et $a l^{21}$ found that walking groups increase physical activity. The results from this study extend these findings by providing evidence of the wide-ranging health benefits of group walking.

Clinicians and therapists may however be asked whether walking in groups has similar health benefits than walking per se or the use of a pedometer, a widely used method of increasing walking. To explore this, the results of the meta-analysis within this study were compared first with meta-analyses of walking and then with pedometers.

In terms of depression, Robertson $\mathrm{et}^{\mathrm{al}} \mathrm{l}^{7}$ in their meta-analysis of walking using a fixed effects model, found a standardised mean effect size of $-0.86(-1.12$ to -0.61$)$, comparable to the effect size of $-0.67(-0.97$ to -0.38$)$ in this review of group walking. In terms of cardiovascular health, a systematic review by Murphy et $a l^{4}$ of walking using a random effects model found statistically significant reductions in body fat, BMI and diastolic blood pressure and increases in $\mathrm{VO}_{2 \max }$. The effects were however of a smaller magnitude than those found in this study; a reduction of diastolic blood pressure of $1.54 \mathrm{~mm} \mathrm{Hg}$ from walking compared with $3.14 \mathrm{~mm} \mathrm{Hg}$ in group walking; a reduction in BMI of $0.2 \mathrm{~kg} / \mathrm{m}^{2}$ compared with $0.7 \mathrm{~kg} / \mathrm{m}^{2}$; and a reduction of body fat of $0.63 \%$ from walking compared with a reduction of $1.31 \%$ in group walking. In addition, Murphy et al did not find a statistically significant reduction in systolic blood pressure $(-1.06 \mathrm{~mm} \mathrm{Hg}, \mathrm{p}=0.316)$ from walking in contrast to the significant reduction in systolic blood pressure $(-3.72 \mathrm{~mm} \mathrm{Hg}, \mathrm{p}<0.001)$ found from group walking in this review. Murphy et $a l^{4}$ stated a relative reduction of $0.8 \%$ in systolic and $2 \%$ in diastolic blood pressure. This is comparable to a previous meta-analysis of walking and resting blood pressure ${ }^{58}$ 
which found a $2 \%$ reduction in systolic and diastolic from walking. In comparison, this review of group walking found reductions of $3 \%$ in systolic $5 \%$ in diastolic blood pressure, representing a greater reduction than those from walking alone. The importance of this difference becomes significant when viewed against findings that a $2 \mathrm{~mm} \mathrm{Hg}$ in diastolic blood pressure can reduce coronary heart disease risk by $6 \%$ and stroke and trans-ischaemic attacks by $15 \% .{ }^{59}$ Further evidence of the importance of this reduction comes from a meta-analysis of prospective studies which suggested that a persistent reduction in average blood pressure by widely practicable methods could avoid large absolute numbers of premature deaths and disabling strokes and a reduction of only $2 \mathrm{~mm} \mathrm{Hg}$ in systolic blood pressure could reduce stroke mortality by $10 \%$ and mortality from vascular causes in a middle-aged population by $7 \% .{ }^{60}$ Outdoor walking groups could be an example of such a practicable method. The second part of this further analysis compared the results from this systematic review of group walking to a systematic review and meta-analysis of pedometers to increase physical activity and improve health outcomes. ${ }^{61}$ Again walking groups were found to have comparable and greater results to those from pedometers in reductions in BMI, systolic and diastolic blood pressure and total cholesterol. This was particularly significant for diastolic blood pressure with the use of pedometers showing a reduction of $-0.3 \mathrm{~mm} \mathrm{Hg}(-0.02$ to -0.46$)$ compared with walking groups $-3.14 \mathrm{~mm} \mathrm{Hg}(-4.15$ to -2.13$)$. It should be noted that the two comparator systematic reviews included outdoor group walking as well as other methods (indoors and solo) in their meta-analysis; within the systematic review of pedometers some of the participants may have walked within a workplace group and additionally people who walk in groups invariably walk by themselves too. Therefore, this further analysis is not a straightforward comparison of nongroup versus group methods but this comparison has provided some evidence that group walking may have benefits to health at least equal to walking with pedometers and walking per se.

\section{Conclusions and meaning of the study for clinicians}

This systematic review with meta-analysis has found that outdoor walking groups have wide-ranging health benefits. With low levels of attrition, high levels of adherence and virtually no adverse effects this study suggests that walking groups could be a practicable intervention, acceptable to patients as a line of treatment with a potential for both physiological and psychological health benefits. It may provide clinicians with evidence of a further effective option to recommend to those patients who would benefit from increasing moderate physical activity.

\section{Unanswered questions and further research}

One study evaluated the results based on three different walk speeds. ${ }^{51}$ Otherwise, there were insufficient studies meeting moderate activity guidelines from which to conduct a subanalysis and suggest any tentative conclusions about effectiveness of walking groups and time or intensity. It may be that effect sizes could be improved by increasing volume and intensity and this important question remains unanswered. A lack of socioeconomic information prevented analysis of the distribution and effects between different social groups confirming concerns raised by Ogilvie et al ${ }^{20}$ that such targeted interventions may be preferentially utilised by better-off groups ${ }^{62}$ and may thereby increase health inequalities. ${ }^{63}$ The issue of equity could be addressed in future research. Additionally, the majority of the studies in this analysis were with people with diagnosed health conditions or cardiovascular disease risk factors; therefore, the potential benefit of walking groups in maintaining good health in healthy populations is not known. Nevertheless, this review has shown that there are wide-ranging health benefits from outdoor walking groups and these appear not to be counterbalanced by an increase in injuries or other adverse side effects.

\section{What are the new findings?}

- Outdoor walking groups have wide-ranging health benefits including reducing blood pressure, body fat, total cholesterol and risk of depression.

- Outdoor walking groups appear to be an acceptable intervention to participants, with high levels of adherence and virtually no adverse effects.

\section{How might it impact on clinical practice in the near future?}

Provides clinicians with evidence of a further effective option to recommend to those patients who would benefit from increasing moderate physical activity.

Acknowledgements This work was partially supported by the Centre for Diet and Activity Research (CEDAR), a UKCRC Public Health Research Centre of Excellence. Funding from the British Heart Foundation, Economic and Social Research Council, Medical Research Council, National Institute for Health Research and the Wellcome Trust, under the auspices of the UK Clinical Research Collaboration, is gratefully acknowledged. The authors thank William Jones, faculty librarian for his help in the search strategy and Dr Toby Smith for helpful advice and comments.

Contributors $\mathrm{SH}$ and $\mathrm{AJ}$ designed the protocol and the search strategy which was executed by SH. SH screened the initial results and extracted data from the primary studies. SH drafted the original manuscript which was critically revised by AJ.

\section{Competing interests None.}

Provenance and peer review Not commissioned; externally peer reviewed.

Open Access This is an Open Access article distributed in accordance with the terms of the Creative Commons Attribution (CC BY 4.0) license, which permits others to distribute, remix, adapt and build upon this work, for commercial use, provided the original work is properly cited. See: http://creativecommons.org/ licenses/by/4.0/

\section{REFERENCES}

1 Naci $H$, loannidis JP. Comparative effectiveness of exercise and drug interventions on mortality outcomes: metaepidemiological study. BMJ 2013;347:f5577.

2 Department of Health. Start active, stay active: a report on physical activity for health from the four home countries' Chief Medical Officers. London: Department of Health, 2011

3 Morris JN, Hardman AE. Walking to health. Sports Med 1997;23:306-32.

4 Murphy MH, Nevill AM, Murtagh EM, et al. The effect of walking on fitness, fatness and resting blood pressure: a meta-analysis of randomised, controlled trials. Prev Med 2007;44:377-85.

5 Lee LL, Watson MC, Mulvaney CA, et al. The effect of walking intervention on blood pressure control: a systematic review. Int J Nurs Stud 2010;47:1545-61.

6 Richardson CR, Newton TL, Abraham JJ, et al. A meta-analysis of pedometer-based walking interventions and weight loss. Ann Fam Med 2008;6:69-77.

7 Robertson R, Robertson A, Jepson R, et al. Walking for depression or depressive symptoms: a systematic review and meta-analysis. Ment Health Phys Act 2012;5:66-75.

8 Hamer M, Chida Y. Walking and primary prevention: a meta-analysis of prospective cohort studies. Br J Sports Med 2008;42:238-43.

9 NHS choices. Change4Life activity planner-health tools. NHS Choices, 2013.

10 SportEngland. Who plays sport? 2013;2013(26/11/13). http://www.sportengland. org/research/who-plays-sport/ 
11 Farrell L, Hollingsworht B, Propper C, et al. The Socioeconomic Gradient in Physical Inactivity in England. 2013. http://www.bristol.ac.uk/cmpo/publications/papers/2013/ wp311.pdf (accessed 26 Nov 2013).

12 Pavey $\mathrm{T}$, Taylor A, Fox $\mathrm{K}$, et al. Effect of exercise referral schemes in primary care on physical activity and improving health outcomes: systematic review and meta-analysis. BMJ 2011;343:d6462.

13 Hillsdon M, Thorogood M, White I, et al. Advising people to take more exercise is ineffective: a randomized controlled trial of physical activity promotion in primary care. Int J Epidemiol 2002;31:808-15.

14 Orrow G, Kinmonth A-L, Sanderson S, et al. Effectiveness of physical activity promotion based in primary care: systematic review and meta-analysis of randomised controlled trials. BMJ 2012;344:e1389.

15 Lamb S, Bartlett $H$, Ashley $A$, et al. Can lay-led walking programmes increase physical activity in middle aged adults? A randomised controlled trial. J Epidemiol Community Health 2002;56:246-52.

16 Macmillan Ramblers. Walking for Health. Secondary Walking for Health. 2013. http://www.walkingforhealth.org.uk/

17 Gusi N, Reyes M, Gonzalez-Guerrero J, et al. Cost-utility of a walking programme for moderately depressed, obese, or overweight elderly women in primary care: a randomised controlled trial. BMC Public Health 2008;8:231.

18 Kwak L, Kremers $S$, Walsh $A$, et al. How is your walking group running? Health Educ 2006;106:21-31.

19 Doughty K. Walking together: the embodied and mobile production of a therapeutic landscape. Health Place 2013;24:140-6.

20 Ogilvie D, Foster C, Rothnie H. Interventions to promote walking: systematic review. BMJ 2007;334:1204-7.

21 Kassavou A, Turner A, French DP. Do interventions to promote walking in groups increase physical activity? A meta-analysis. Int J Behav Nutr Phys Act 2013;10:18.

22 Higgins J, Green S. Cochrane handbook for systematic reviews of interventions version 5.1. O [updated March 2011]. The Cochrane Collaboration, 2011.

23 PROSPERO. International Prospective Register of Systematic Reviews: University of York, Centre for reviews and dissemination. 2013.

24 Liberati A, Altman DG, Tetzlaff J, et al. The PRISMA statement for reporting systematic reviews and meta-analyses of studies that evaluate healthcare interventions: explanation and elaboration. BMJ 2009;339:b2700.

25 Moher D, Liberati A, Tetzlaff J, et al. Preferred reporting items for systematic reviews and meta-analyses: the PRISMA statement. BMJ 2009;339:b2535.

26 Deeks J, Dinnes J, D'Amico R, et al. Evaluating non-randomised intervention studies. Health Technol Assess (Rockv) 2003;7:186.

27 Sterne JA, Egger M. Funnel plots for detecting bias in meta-analysis: guidelines on choice of axis. J Clin Epidemiol 2001;54:1046-55.

28 Review Manager(RevMan) [computer program], The Nordic Cochrane Centre, The Cochrane Collaboration 2012 [program]. Version 5.2 version. Copenhagen.

29 Brosseau L, Wells GA, Kenny GP, et al. The implementation of a community-based aerobic walking program for mild to moderate knee osteoarthritis: a knowledge translation randomized controlled trial: part II: clinical outcomes. BMC Public Health 2012;12:1073-3.

30 Callahan LF, Shreffler JH, Altpeter M, et al. Evaluation of group and self-directed formats of the arthritis foundation's Walk With Ease program. Arthritis Care Res 2011;63:1098-107.

31 Holmberg SK. Evaluation of a clinical intervention for wanderers on a geriatric nursing unit. Arch Psychiatr Nurs 1997;11:21-8.

32 Thomas DW, Glogoski C, Johnson J. The effect of a supervised walking program on wandering among residents with Dementia. Act Adapt Aging 2006;30:1-13.

33 Van Uffelen JGZ, Chin APMJM, Hopman-Rock M, et al. The effect of walking and vitamin B supplementation on quality of life in community-dwelling adults with mild cognitive impairment: a randomized, controlled trial. Qual Life Res 2007;16:1137-46.

34 Fritz $\mathrm{T}$, Wandell $\mathrm{P}$, Aberg $\mathrm{H}$, et al. Walking for exercise-does three times per week influence risk factors in type 2 diabetes? Diabetes Res Clin Pract 2006;71:21-7.

35 Negri C, Bacchi E, Morgante S. Supervised walking groups to increase physical activity in type 2 diabetic patients. Diabetes Care 2010;33:2333-5.

36 O'Halloran PD. Mood changes in weeks 2 and 6 of a graduated group walking program in previously sedentary people with type 2 diabetes. Aust J Prim Health 2007;13:68-73.

37 Bjersing JL, Dehlin $M$, Erlandsson $M$, et al. Changes in pain and insulin-like growth factor 1 in fibromyalgia during exercise: the involvement of cerebrospinal inflammatory factors and neuropeptides. Arthritis Res Ther 2012;14:R162.
38 Kayo AH, Peccin MS, Sanches CM, et al. Effectiveness of physical activity in reducing pain in patients with fibromyalgia: A blinded randomized clinical trial. Rheumatol Int 2012;32:2285-92.

39 Mannerkorpi K, Nordeman L, Cider $\AA$, et al. Does moderate-to-high intensity Nordic walking improve functional capacity and pain in fibromyalgia? A prospective randomized controlled trial. Arthritis Res Ther 2010;12:R189.

40 Brandon LJ, Elliott-Lloyd MB. Walking, body composition, and blood pressure dose-response in African American and White women. Ethn Dis 2006;16:675-81.

41 Figard-Fabre H, Fabre N, Leonardi A, et al. Efficacy of Nordic walking in obesity management. Int J Sports Med 2011;32:407-14.

42 Hinkleman LL, Nieman DC. The effects of a walking program on body composition and serum lipids and lipoproteins in overweight women. J Sports Med Phys Fitness 1993;33:49-58.

43 Moss S. Changes in coronary heart disease risk profile of adults with intellectual disabilities following a physical activity intervention. J Intellect Disabil Res 2009;53:735-44.

44 O'Hara RB, Baer JT, Pohlman RL, et al. The effect of a walking program on blood pressure response in African-American women. ACSM Health Fitness $J$ 2000:4:20-4.

45 Armstrong $\mathrm{K}$, Edwards $\mathrm{H}$. The effectiveness of a pram-walking exercise programme in reducing depressive symptomatology for postnatal women. Int J Nurs Pract 2004;10:177-94.

46 Dallocchio C, Arbasino C, Klersy C, et al. The effects of physical activity on psychogenic movement disorders. Mov Disord 2010;25:421-5.

47 Legrand FD, Mille CR. The effects of 60 minutes of supervised weekly walking (in a single vs. 3-5 session format) on depressive symptoms among older women: Findings from a pilot randomized trial. Ment Health Phys Act 2009;2:71-5.

48 McDevitt J, Robinson N, Forest D. A group-based walking program at a psychiatric rehabilitation center. Psychiatr Serv 2005;56:354-5.

$49 \mathrm{Ng} \mathrm{F}$, Dodd S, Jacka FN, et al. Effects of a walking program in the psychiatric in-patient treatment setting: a cohort study. Health Promot J Austr 2007;18:39-42.

50 Reuter I, Mehnert $S$, Leone $P$, et al. Effects of a flexibility and relaxation programme, walking, and nordic walking on parkinson's disease. J Aging Res 2011;2011:232473.

51 Duncan JJ, Gordon NF, Scott CB. Women walking for health and fitness: how much is enough? JAMA 1991;266:3295-9.

52 Silverthorn $\mathrm{KH}$, Hornak JE. Beneficial effects of exercise on aerobic capacity and body composition in adults with Prader-Willi syndrome. Am J Ment Retard 1993:97:654-8

53 Moore-Harrison TL, Speer EM, Johnson FT, et al. The effects of aerobic training and nutrition education on functional performance in low socioeconomic older adults. J Geriatr Phys Ther 2008;31:18-23.

54 Isaacs AJ, Critchley JA, Tai SS, et al. Exercise Evaluation Randomised Trial (EXERT): a randomised trial comparing GP referral for leisure centre-based exercise, community-based walking and advice only. Health Technol Assess 2007;11:1-165, iii-iv.

55 Cyarto EV, Brown WJ, Marshall AL, et al. Comparison of the effects of a home-based and group-based resistance training program on functional ability in older adults. Am J Health Promot 2008;23:13-7.

56 Cox KL, Burke V, Beilin LJ, et al. Blood pressure rise with swimming versus walking in older women: the Sedentary Women Exercise Adherence Trial 2 (SWEAT 2) J Hypertens 2006;24:307-14.

57 Fantin F, Rossi A, Morgante S, et al. Supervised walking groups to increase physical activity in elderly women with and without hypertension: effect on pulse wave velocity. Hypertens Res 2012;35:988-93.

58 Kelley GA, Kelley KS, Tran ZV. Walking and resting blood pressure in adults: a meta-analysis. Prev Med 2001;33:120-7.

59 Cook NR, Cohen J, Hebert PR, et al. Implications of small reductions in diastolic blood pressure for primary prevention. Arch Intern Med 1995;155:701.

60 Lewington S, Clarke R, Qizilbash N, et al. Age-specific relevance of usual blood pressure to vascular mortality: a meta-analysis of individual data for one million adults in 61 prospective studies. Lancet 2002;360:1903-13.

61 Bravata DM, Smith-Spangler C, Sundaram V, et al. Using pedometers to increase physical activity and improve health: a systematic review. JAMA 2007;298:2296-304.

62 Tudor Hart J. The inverse care law. Lancet 1971;297:405-12.

63 Marmot M, Allen J, Goldblatt P, et al. Fair society, healthy lives: Strategic review of health inequalities in England post-2010. 2010. 